\title{
UMA FORMULAÇÃO LMI PARA A ANÁLISE DE ESTABILIDADE COM FUNÇÕES DE LYAPUNOV DO TIPO LUR'E-PERSIDSKII
}

\author{
M. C. de Oliveira* \\ mauricio@mechanics.ucsd.edu \\ L. $\mathrm{Hsu}^{\dagger}$ \\ liu@coep.ufrj.br \\ J. C. Geromel* \\ geromel@dt.fee.unicamp.br \\ *Departamento de Telemática, \\ Faculdade de Engenharia Elétrica e de Computação, UNICAMP, \\ CP 6101, 13081-970, Campinas, SP. \\ ${ }^{\dagger}$ Programa de Engenharia Elétrica, COPPE-UFRJ, \\ CP 68504, 21945-970, Rio de Janeiro, RJ.
}

\begin{abstract}
In this paper we propose an unified construction of Lyapunov functions based on Lur'e type functions that allows us to go from Persidskii's to pure quadratic functions. Such Lyapunov functions allows the establishment of the absolute stability of nonlinear systems with nonlinearities belonging to given sectors. The sectors can be finite or infinite. An stability criterion is formulated in terms of Linear Matrix Inequalities (LMI). The parameters that define the sectors are present in the given LMI and are made available for optimization, leading to several optimization problems that are able to determine the available robustness levels for several sector configurations.
\end{abstract}

\section{RESUMO}

Neste artigo propomos uma método unificado para construção de funções de Lyapunov a partir de funções do tipo Lur'e que contém como casos particulares as funções do tipo Persidskii e as funções puramente quadrá-

\footnotetext{
Artigo submetido em 20/12/00

1a. Revisão em 07/01/01; 2a. Revisão em 01/02/02

Aceito sob recomendação do Ed. Cons. Prof. Dr. José Roberto Castilho Piqueira
}

ticas. Estas funções de Lyapunov permitem estabelecer um critério para a estabilidade absoluta de sistemas não lineares com não linearidades pertencentes a setores, sejam eles finitos ou infinitos. O critério de estabilidade é formulado por meio de desigualdades matriciais lineares (LMIs) nas quais os parâmetros que definem os setores estão disponíveis para otimização. Esta propriedade nos permite definir problemas de otimização para determinação dos níveis de robustez para diversas configurações dos setores.

KEYWORDS: Estabilidade Absoluta, Desigualdades Matriciais Lineares, Critério do Círculo, Critério de Popov

\section{INTRODUÇÃO}

Neste artigo estamos interessados em estudar a estabilidade de sistemas descritos por equações diferenciais no seguinte formato

$$
\dot{x}_{i}=\sum_{j=1}^{n} a_{i j} f_{j}\left(x_{j}\right), \quad i=1, \ldots, n,
$$

em que $x \in \Re^{n}$ é o vetor de estado e no qual as funções $f_{j}: \Re \rightarrow \Re$ não são necessariamente lineares. Podemos nos referir a este tipo de sistema de maneira mais 
compacta por meio da notação

$$
\dot{x}=A f(x),
$$

em que $A:=\left(a_{i j}\right)$ e $f:=\left(f_{1}, \ldots, f_{n}\right)$.

Dizemos que a matriz $A$ é diagonalmente estável se existe uma matriz $P$ diagonal e definida positiva que soluciona a desigualdade de Lyapunov

$$
A^{T} P+P A<0 .
$$

Neste caso, sabemos que a origem é um ponto de equilíbrio estável do sistema (1) para todas as funções $f_{j}$ pertencentes à classe (Persidskii, 1969)

$$
\mathcal{F}:=\{f: \Re \rightarrow \Re, \quad f(\tau) \tau>0, f(0)=0\}
$$

O fato que está por trás deste resultado é a definição da seguinte função de Lyapunov

$$
V(x):=\sum_{i=1}^{n} p_{i i} \int_{0}^{x_{i}} f(\tau) d \tau
$$

chamada aqui função de Persidskii. Se a matriz diagonal e definida positiva $P:=\left(p_{i i}\right)$ soluciona a equação (3) então

$$
\dot{V}(x):=f(x)^{T}\left(A^{T} P+P A\right) f(x)<0
$$

ao longo de todas as trajetórias do sistema (1).

Sistemas descritos pela equação diferencial (2) cujas funções em $f(\cdot)$ pertencem à classe $\mathcal{F}$ têm um papel importante no estudo da estabilidade de sistemas não lineares (Kaszkurewicz e Bhaya, 1993). Esta classe de sistemas pode também representar sistemas com perturbações sobre o vetor de estado, os quais se fazem presentes em diversas áreas, conforme (Kaszkurewicz e Bhaya, 1993; Kaszkurewicz e Hsu, 1979; Chu e Glover, 1999). Aplicações recentes desta classe de sistemas incluem o estudo da estabilidade de redes neurais de Hopfield (Kaszkurewicz e Bhaya, 1994) e sistemas com elementos sujeitos a saturação (Albertini e D'Alessandro, 1996). Como também notado por outros autores (Kaszkurewicz e Bhaya, 1993; Chu e Glover, 1999), a importância desta classe de sistemas se torna ainda maior se percebemos que um sistema na forma

$$
\dot{y}=f(A y),
$$

em que $f:=\left(f_{1}, \ldots, f_{n}\right), f_{i} \in \mathcal{F}, i=1, \ldots, n$, pode ser também descrito por meio da equação (2). De fato, se a matriz $A$ for Hurwitz, isto é, se todos os autovalores de $A$ se encontrarem no semiplano esquerdo do plano complexo, a transformação de variáveis $x=A y$ é capaz de colocar o sistema (7) na forma (2).
Em alguns casos, a classe de funções não lineares $\mathcal{F}$, definida em (4), e a função de Persidskii associada (5) podem ser muito restritivas. Alguns autores têm tentado incorporar certa flexibilidade a este contexto impondo que a matriz $P$ seja apenas diagonal dominante, ao invés de diagonal. Desta maneira, alguns resultados menos conservadores puderam ser obtidos para sistemas com perturbações paramétricas (Kaszkurewicz e Bhaya, 1993) e com perturbações repetidas (Chu e Glover, 1999).

Nosso objetivo neste artigo é prover uma maneira simples e flexível de estudar a estabilidade de sistemas na forma (1) com relação à classe de funções

$$
\mathcal{F}(\gamma, \delta):=\left\{f: \Re \rightarrow \Re ; \gamma \tau^{2}<f(\tau) \tau<\delta \tau^{2}, f(0)=0\right\}
$$

definidas para valores no intervalo $0 \leq \gamma \leq 1 \leq \delta$. Devido à interpretação geométrica desta definição, dizemos que uma função pertencente a $\mathcal{F}(\gamma, \delta)$ encontra-se no setor $\mathcal{F}(\gamma, \delta)$. Casos particulares de interesse podem ser gerados variando-se os valores dos parâmetros $\gamma$ e $\delta$. Por exemplo, o conjunto de funções $\mathcal{F}(1,1)=\{f(\tau)=\tau\}$ contém apenas a função identidade; a classe $\mathcal{F}(\gamma, \delta)$ torna-se equivalente à classe $\mathcal{F}$ definida em (4) à medida em que $(\gamma, \delta) \rightarrow(0, \infty)$.

Para que possamos obter estes resultados, a restrição de que $P$ seja uma matriz diagonal deve ser relaxada. Conseguimos isto lançando mão de funções do tipo Lur'e, que permitem incorporar os parâmetros $\gamma$ e $\delta$ nas condições para estabilidade. Estas condições são apresentadas como um conjunto de desigualdades matriciais lineares (LMI) (Boyd et al., 1994), o que torna possível definir problemas de otimização para determinação do domínio de robustez em termos dos parâmetros $\gamma$ e $\delta$. Uma vez que parâmetros $\gamma_{i}$ e $\delta_{i}, i=1, \ldots, n$ são definidos individualmente para cada perturbação $f_{i}$, os resultados apresentados são aplicáveis também a sistemas parcialmente perturbados, isto é, sistemas nos quais apenas uma parte do vetor de estado é realimentado por meio de uma função não linear. Encontra-se este tipo de sistema, por exemplo, no estudo do problema de controle de freqüência de sistemas de potência (Hsu e Salgado, 1980; Pai, 1981).

Uma outra classe de sistemas que se encontra ao alcance dos métodos apresentados é a classe dos sistemas com perturbações do vetor de estado do tipo multiplicativa, como em

$$
\dot{x}_{i}=\sum_{j=1}^{n} a_{i j}\left(1+\sigma_{j}\right) x_{j}, \quad j=1, \ldots, n,
$$

O valor da perturbação $\sigma_{j} x_{j}$ depende da amplitude do sinal $x_{j}$ e o parâmetro $\sigma_{j}$ pode ser interpretado 
como uma medida da relação sinal/ruído, o que faz com que sistemas na forma (9) nos quais $\sigma_{j}$ seja limitado constituam um caso particular de sistemas com relação sinal/ruído finita (FSN) estudados, por exemplo, em (Thygesen e Skelton, 1995). Definindo-se os limites $\left|\sigma_{j}\right| \leq \xi_{j}, 0<\xi_{j}<1$, a estabilidade do sistema (9) pode ser verificada testando-se a estabilidade do sistema (2) com relação ao setor $\mathcal{F}\left(1-\xi_{j}, 1+\xi_{j}\right)$.

\subsection{Notação}

O símbolo $\left({ }^{T}\right)$ indica transposição tanto para matrizes quanto para vetores. A notação $P>0$ significa que a matriz $P$ é simétrica $\left(P=P^{T}\right)$ e definida positiva. Para matrizes quadradas, o operador trace $(\cdot)$ denota o traço de $(\cdot)$. A matriz identidade e a matriz nula são grafadas, respectivamente, $\mathbf{I}$ e $\mathbf{0}$.

\section{ANÁLISE DE ESTABILIDADE}

Abrimos esta seção definindo a seguinte candidata a função de Lyapunov

$$
V(x):=x^{T} W x+\sum_{i=1}^{n} p_{i i} \int_{0}^{x_{i}} f(\tau) d \tau,
$$

em que $W>0$ e $P:=\left(p_{i i}\right)>0$, por nós chamada função de Lur'e. No contexto do estudo da estabilidade absoluta de sistemas não lineares, a função de Lur'e encontrase associada ao critério de Popov ao passo que formas quadráticas puras se encontram associadas ao critério do círculo. Para uma descrição mais completa destes conceitos veja, por exemplo, (Khalil, 1996).

A derivada em relação ao tempo da função (10) ao longo de qualquer trajetória do sistema (2) é dada por

$$
\dot{V}(x)=2 f^{T} A^{T} W x+f^{T}\left(A^{T} P+P A\right) f .
$$

Os setores definidos em (8) implicam que

$$
\left(f_{i}-\gamma_{i} x_{i}\right)\left(f_{i}-\delta_{i} x_{i}\right)<0, \quad i=1, \ldots, n
$$

e, portanto,

$$
f^{T} f-f^{T}(\Gamma+\Delta) x+x^{T} \Gamma \Delta x<0,
$$

em que $\Gamma:=\operatorname{diag}\left(\gamma_{1}, \ldots, \gamma_{n}\right)$ e $\Delta:=\operatorname{diag}\left(\delta_{1}, \ldots, \delta_{n}\right)$.

Combinando as desigualdades (11) e (13) podemos concluir que, caso exista uma matriz diagonal e definida positiva $Q:=\left(q_{i i}\right)$ de tal forma que

$$
\dot{V}(x)<f^{T} Q f-f^{T}(\Gamma+\Delta) Q x+x^{T} \Gamma \Delta Q x,
$$

então a derivada em relação ao tempo da função $V(x)$ ao longo de qualquer trajetória do sistema (2) que satisfaça a condição de setor, definida em (8), é negativa.
Conseqüentemente, a origem é um ponto de equilíbrio estável para o sistema. A condição (14) pode ser descrita por meio das seguintes LMIs

$$
\begin{array}{cc}
{\left[\begin{array}{cc}
A^{T} P+P A-Q & A^{T} W+\frac{1}{2}(\Gamma+\Delta) Q \\
W A+\frac{1}{2} Q(\Gamma+\Delta) & -\Gamma \Delta Q
\end{array}\right]<0} \\
W>0, \quad P>0 &
\end{array}
$$

Com a discussão acima, acabamos de provar o seguinte teorema.

Teorema 1 A origem é um ponto de equilíbrio estável do sistema $\dot{x}=A f(x)$ para toda função $f_{i} \in$ $\mathcal{F}\left(\gamma_{i}, \delta_{i}\right), i=1, \ldots, n$ se existirem matrizes diagonais $e$ definidas positivas $P$ e $Q$, e uma matriz simétrica e definida positiva $W$ de tal forma que as LMIs (15) sejam simultaneamente factiveis.

O teste de estabilidade representado pelas LMIs (15) é genérico o bastante para conter diversos resultados atualmente disponíveis na literatura. O objetivo das próximas seções é avaliar esta capacidade com respeito a diferentes configurações dos setores $\mathcal{F}\left(\gamma_{i}, \delta_{i}\right), i=1, \ldots, n$.

Antes de procedermos a estas análises, cabe ressalvar que o teste apresentado no Teorem 1 é apenas uma condição suficiente de estabilidade, podendo levar, em alguns casos, a análises conservadoras. Esta limitação, não impede porém que observemos melhora significativa em relação aos critérios atualmente existentes de estabilidade para este tipo de sistema (veja exemplos na Seção 4).

\subsection{Sistemas puramente lineares}

Nesta seção, mostramos que o Teorema 1 não é conservador quando aplicado à análise de sistemas puramente lineares, isto é, sistemas na forma $\dot{x}=A x$. Neste caso, a configuração apropriada dos setores $\mathcal{F}\left(\gamma_{i}, \delta_{i}\right), i=$ $1, \ldots, n$, é ditada pela escolha particular das matrizes $\Gamma=\Delta=\mathbf{I}$.

Lema 2 Se existir uma matriz simétrica e definida positiva $W$ que satisfaça a desigualdade

$$
A^{T} W+W A<0,
$$

o sistema linear $\dot{x}=A x$ é dito assintoticamente estável. Neste caso, a matriz $W$ definida acima, a matriz $P \rightarrow 0^{+} \mathbf{I}$ e uma matriz diagonal e definida positiva $Q$ suficientemente grande satisfazem o Teorema $1 \mathrm{com}$ $\Gamma=\Delta=\mathbf{I}$ e a função de Lyapunov (10) reduz-se a uma função puramente quadrática. 
Prova: Como (16) é estritamente factível, podemos sempre calcular uma matriz $P \rightarrow 0^{+} \mathbf{I}$ e uma matriz diagonal e definida positiva $Q$ suficientemente grande de tal forma que

$$
A^{T} W+W A+A^{T} P+P A+A^{T} W Q^{-1} W A<0 .
$$

Isto conclui esta prova uma vez que a desigualdade acima é o complemento de Schur (Boyd et al., 1994) da primeira LMI em (15) caso $\Gamma+\Delta=2 \mathbf{I}$ e $\Gamma \Delta=\mathbf{I}$.

\subsection{Não linearidades no primeiro e no ter- ceiro quadrantes}

Uma segunda importante configuração dos setores $\mathcal{F}\left(\gamma_{i}, \delta_{i}\right), i=1, \ldots, n$, está associada à escolha das matrizes $\Gamma \rightarrow 0^{+} \mathbf{I}$ e $\Delta \rightarrow \infty \mathbf{I}$. Neste caso, todos os setores $\mathcal{F}\left(\gamma_{i}, \delta_{i}\right)$ se tornam equivalentes ao setor $\mathcal{F}$, definido em (4). Em relação a esta configuração, mostramos por meio do próximo lema que o Teorema 1 contém o Teorema de Persidskii (Persidskii, 1969) como um caso particular.

Lema 3 Se existir uma matriz diagonal e definida positiva $P$ que satisfaça a desigualdade

$$
A^{T} P+P A<0,
$$

a origem é um ponto de equilíbrio estável do sistema (1) para toda função $f_{j} \in \mathcal{F}$, definida em (4). Neste caso, a matriz $P$ definida acima, a matriz $W \rightarrow 0^{+} \mathbf{I}$ e uma matriz diagonal e definida positiva $Q$ suficientemente pequena satisfazem o Teorema $1 \mathrm{com} \Gamma \rightarrow 0^{+} \mathbf{I} e \Delta \rightarrow \infty \mathbf{I}$ e a função de Lyapunov (10) reduz-se a uma função de Persidskii.

Prova: Como (17) é estritamente factível, para todo escalar $\alpha>0$ podemos calcular uma matriz diagonal e definida positiva $Q$ suficientemente pequena de tal forma que a desigualdade

$$
A^{T} P+P A+\frac{\left(\alpha^{-1}+\alpha\right)^{2}-4}{4} Q<0
$$

seja factível. A desigualdade acima é exatamente o complemento de Schur da primeira LMI em (15) com $W \rightarrow 0^{+} \mathbf{I}, \Gamma=\alpha \mathbf{I}$ e $\Delta=\alpha^{-1} \mathbf{I}$. Concluímos a prova observando que isto é verdade para qualquer valor de $\alpha>0$, em particular, para $\alpha \rightarrow 0^{+}$.

Uma outra condição de estabilidade para o sistema (1) com não linearidades no setor $\mathcal{F}$, definido em (4), foi obtida em (Geromel et al., 1998). Vale a pena ressaltarmos que a metodologia empregada em (Geromel et al., 1998) é completamente distinta da descrita no presente artigo. O próximo lema mostra que o Teorema 1 também contém este resultado.

Lema 4 Se existir uma matriz simétrica e definida positiva $W$ que satisfaça a desigualdade

$$
A^{T} W+W A<0, \quad A^{T} W \text { diagonal }
$$

a origem é um ponto de equilíbrio estável do sistema (1) para toda função $f_{j} \in \mathcal{F}$, definida em (4). Neste caso, a matriz $W$ definida acima, a matriz $P \rightarrow 0^{+} \mathbf{I}$ e uma matriz diagonal e definida positiva $Q$ suficientemente pequena satisfazem o Teorema 1 com $\Gamma \rightarrow 0^{+} \mathbf{I} e \Delta \rightarrow \infty \mathbf{I}$ e a função de Lyapunov (10) reduz-se à uma função puramente quadrática.

Prova: A partir de uma solução factível $W$ para a desigualdade (18) e um escalar $\alpha>0$ definimos a matriz diagonal e definida positiva

$$
Q:=-\frac{2}{\alpha+\alpha^{-1}} A^{T} W>0 .
$$

Estas matrizes e $P \rightarrow 0^{+} \mathbf{I}$ constituem uma solução factível para as LMI (15) com $\Gamma=\alpha \mathbf{I}$ e $\Delta=\alpha^{-1} \mathbf{I}$. Concluímos assim esta prova pois isto é verdade para todo valor de $\alpha>0$, em particular para $\alpha \rightarrow 0^{+}$.

O Teorema 1 e os lemas acima enunciados revelam a ligação entre a análise de estabilidade obtida a partir de uma função de Lyapunov puramente quadrática, de uma função de Lur'e (10), e de uma função de Persidskii (5). A obtenção destas relações no contexto da análise de estabilidade de sistemas na forma (1) é bastante interessante uma vez que, segundo a teoria de estabilidade absoluta, estas funções estão associadas, respectivamente, ao critério do círculo, ao critério de Popov e ao teorema de Persidskii. Por meio de uma única condição descrita pelas LMIs (15) fomos capazes de tratar todos estes problemas de maneira unificada.

Antes de prosseguirmos, notemos que o resultado expresso no Lema 4 é sujeito a uma interpretação alternativa por meio do conceito de positividade real (Khalil, 1996).

Corolário 5 As seguintes afirmações são equivalentes:

a) Existe uma matriz simétrica e definida positiva $W$ de tal forma que a desigualdade (18) seja factível. 
b) Existe uma matriz diagonal e definida positiva $Q$ de tal forma que a função de transferência

$$
H(s):=-Q(s \mathbf{I}-A)^{-1} A
$$

seja positiva real estrita (SPR).

Prova: Uma função de transferência genérica $C(s \mathbf{I}-A) B$ é dita SPR se existe uma matriz simétrica e definida positiva $W$ de tal forma que

$$
A^{T} W+W A<0, \quad B^{T} W=C
$$

Se substituirmos os valores de $A, B$ e $C$ presentes em $H(s)$ na expressão acima obtemos

$$
A^{T} W+W A<0, \quad A^{T} W=-Q<0
$$

que é completamente equivalente a (18).

\subsection{0 caso puramente quadrático}

Uma releitura do resultado (Geromel et al., 1998) através do Lema 4 nos leva à seguinte conclusão: é possivel estudar a estabilidade de sistemas com perturbações no setor $\mathcal{F}$ apenas com a ajuda de uma função de Lyapunov puramente quadrática. Podemos obter a generalização deste resultado para o setor $\mathcal{F}(\gamma, \delta)$ simplesmente fazendo com que a matriz $P$ seja nula em (10), o que torna a função de Lur'e a uma função puramente quadrática. Conseqüentemente, as LMIs (15) reduzem-se a

$$
\begin{aligned}
& {\left[\begin{array}{cc}
-Q & A^{T} W+\frac{1}{2}(\Gamma+\Delta) Q \\
W A+Q(\Gamma+\Delta) \frac{1}{2} & -\Gamma \Delta Q
\end{array}\right]<0,} \\
& W>0 .
\end{aligned}
$$

Assim, podemos estabelecer o seguinte corolário ao Teorema 1.

Corolário 6 A origem é um ponto de equilíbrio estável do sistema $\dot{x}=A f(x)$ para toda função $f_{i} \in$ $\mathcal{F}\left(\gamma_{i}, \delta_{i}\right), i=1, \ldots, n$ se existirem uma matriz diagonal e definida positiva $Q$ e uma matriz simétrica e definida positiva $W$ de tal forma que as LMIs (19) sejam simultaneamente factiveis.

Ademais, o Corolário 5 nos indica que, no caso puramente quadrático, podemos tentar obter relações entre as novas condições apresentadas neste artigo e o conceito de positividade real. No restante desta seção, procuraremos obter representações equivalentes para o Corolário 6 em termos dos conceitos de positividade real e norma
$H_{\infty}$ (Colaneri et al., 1997). Para tal, reescrevamos as LMIs (19) na forma equivalente

$$
\begin{aligned}
& {\left[\begin{array}{ccc}
\frac{1}{2}(\Gamma+\Delta) A^{T} W+(\bullet)^{T} & W A & \frac{1}{2}(\Delta-\Gamma) Q \\
A^{T} W & -Q & \mathbf{0} \\
Q(\Delta-\Gamma) \frac{1}{2} & \mathbf{0} & -Q
\end{array}\right]<0,} \\
& W>0
\end{aligned}
$$

em que $(\bullet)$ representa a matriz da primeira parcela da expressão onde se encontra. A equivalência entre (19) e (20) é estabelecida da seguinte maneira: tomando o complemento de Schur de (19) obtemos

$$
\begin{aligned}
\frac{1}{2}(\Gamma+\Delta) A^{T} W+W A(\Gamma+\Delta) \frac{1}{2} \\
+W A Q^{-1} A^{T} W+\frac{1}{4}(\Delta-\Gamma)^{2} Q<0 .
\end{aligned}
$$

Se nos lembrarmos de que $\Delta>\Gamma$ e usarmos o complemento de Schur mais uma vez recuperamos (20). Esta condição nos permite identificar a seguintes equivalências.

Lema 7 As seguintes afirmações são equivalentes:

a) Existem uma matriz diagonal e definida positiva $Q$ e uma matriz simétrica e definida positiva $W$ de tal forma que o Corolário 6 seja satisfeito.

b) Existe uma matriz diagonal e definida positiva $Q$ de tal forma que a função de transferência

$$
\begin{aligned}
& H(s):=\left[\begin{array}{c}
\mathbf{0} \\
\frac{1}{2} Q(\Gamma-\Delta)
\end{array}\right] . \\
& {\left[s \mathbf{I}-\frac{1}{2} A(\Gamma+\Delta)\right]^{-1}\left[\begin{array}{ll}
A & \mathbf{0}
\end{array}\right]+\frac{1}{2}\left[\begin{array}{ll}
Q & \mathbf{0} \\
\mathbf{0} & Q
\end{array}\right]}
\end{aligned}
$$

seja positiva real estrita e estendida(ESPR).

c) Existe uma matriz diagonal e definida positiva $Q$ de tal forma que a função de transferência

$$
\begin{aligned}
H(s) & :=\frac{1}{2} Q^{\frac{1}{2}}(\Delta-\Gamma) . \\
& {\left[s \mathbf{I}-\frac{1}{2} A(\Gamma+\Delta)\right]^{-1} A Q^{-\frac{1}{2}} }
\end{aligned}
$$

tenha norma $H_{\infty}$ menor do que um.

Prova: Inicialmente provamos que o ítem a) é equivalente ao ítem $b$ ). Uma função de transferência genérica

$$
C(s \mathbf{I}-A)^{-1} B+D
$$


é dita ESPR se existe uma matriz simétrica e definida positiva $W$ de tal forma que

$$
\left[\begin{array}{cc}
A^{T} W+W A & W B-C^{T} \\
B^{T} W-C & -D-D^{T}
\end{array}\right]<0
$$

Esta LMI é conhecida pelo nome de "lema da positividade real" (Positive Real Lemma) (Colaneri et al., 1997). Se substituirmos os valores de $A, B, C$ e $D$ em (21) na expressão acima obtemos (20).

Provemos agora a equivalência entre $a$ ) e $c$ ). Uma função de transferência genérica

$$
C(s \mathbf{I}-A)^{-1} B
$$

tem norma $H_{\infty}$ menor do que um se, e somente se, existe uma matriz simétrica e definida positiva $W$ de tal forma que

$$
\left[\begin{array}{ccc}
A^{T} W+W A & W B & C^{T} \\
B^{T} W & -\mathbf{I} & \mathbf{0} \\
C & \mathbf{0} & -\mathbf{I}
\end{array}\right]<0 .
$$

Esta LMI é conhecida pelo nome de "lema da limitação real" (Bouded Real Lemma) (Colaneri et al., 1997). Se substituirmos os valores de $A, B$ e $C$ em (22) na expressão acima obtemos $(\operatorname{com}(\bullet)$ significando o mesmo que em (20))

$$
\begin{aligned}
& {\left[\begin{array}{ccc}
\frac{1}{2}(\Gamma+\Delta) A^{T} W+(\bullet) & W A Q^{-\frac{1}{2}} & \frac{1}{2}(\Delta-\Gamma) Q^{\frac{1}{2}} \\
Q^{-\frac{1}{2}} A^{T} W & -\mathbf{I} & \mathbf{0} \\
Q^{\frac{1}{2}}(\Delta-\Gamma) \frac{1}{2} & \mathbf{0} & -\mathbf{I}
\end{array}\right]} \\
& <0
\end{aligned}
$$

que, multiplicado de ambos os lados pela matriz diagonal $T:=\operatorname{diag}\left(\mathbf{I}, Q^{\frac{1}{2}}, Q^{\frac{1}{2}}\right)$, se transforma em (20).

Notemos que a matriz $Q$ se presta ao papel de scaling nas condições $b$ ) e $c$ ) do Lema 7 .

\section{OTIMIZANDO OS SETORES}

Uma das propriedades mais interessantes dos resultados deste artigo é que os parâmetros do setor $\mathcal{F}(\gamma, \delta)$, definido em (8), se encontram presentes nas condições de estabilidade. Conseqüentemente, e conforme explicado mais adiante, torna-se possível definir problemas de otimização nos quais estes parâmetros possam ser otimizados visando determinar os maiores domínios de robustez disponíveis. Diversas estratégias são possíveis, dependendo do tipo de setor considerado.

Suponhamos que os setores $\mathcal{F}\left(\gamma_{i}, \delta_{i}\right), i=1, \ldots, n$, modelem efeitos de saturação no estado como, por exemplo,

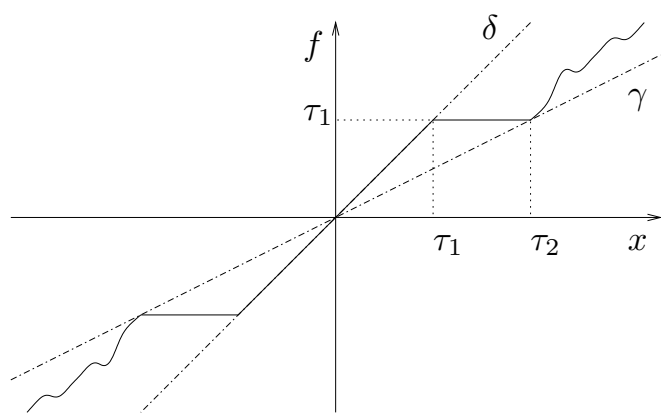

Figura 1: Curva de saturação

em (Albertini e D'Alessandro, 1996). Neste caso, podemos imaginar que as funções $f_{j}$ se pareçam com a linha sólida mostrada na Figura 1. O valor da derivada $\delta$ é suposto conhecido e, sem perda de generalidade, é considerado igual a um. O problema de otimização aqui proposto é o de minimizar $\gamma$. A razão para isto é que quanto menor o valor de $\gamma$, maior o valor de $\tau_{2}$, isto é, maior o limite de robustez em relação à saturação no nível $\tau_{1}$. Se pudermos obter $\gamma$ arbitrariamente próximo ao zero, o sistema torna-se robusto à saturação em qualquer nível. Notemos que o valor $\tau_{2}$ pode ser entendido como um limite prático ao máximo valor do estado considerado. Apenas para efeito de simplificação da exposição, supomos que todas as não linearidades são do mesmo tipo. Neste caso, o problema de minimização de $\gamma$ pode ser descrito, por exemplo, atribuindo $\Delta=\mathbf{I}$ e introduzindo a mudança de variáveis

$$
V:=\Gamma Q .
$$

Assim, a partir de (15), obtemos o programa

$$
\begin{aligned}
& \min \quad \lambda, \\
& \text { t.q. } \quad \lambda Q>V, \\
& {\left[\begin{array}{cc}
A^{T} P+P A-Q & A^{T} W+\frac{1}{2}(V+Q) \\
W A+\frac{1}{2}(V+Q) & -V
\end{array}\right]<0,} \\
& W>0, \\
& Q>0, \quad V>0, \quad P>0 \quad \text { diagonais. }
\end{aligned}
$$

A idéia por trás da definição deste problema é que $\lambda \mathbf{I}>\Gamma \mathrm{e}$, portanto, minimizando $\lambda$ estaremos também minimizando $\Gamma$. Notemos que $\lambda$ pode ser entendido como o máximo autovalor do feixe diagonal $(Q, V)$, o que caracteriza este problema como um problema de otimização quase convexo, cuja solução ótima global pode ser obtida por meio de algoritmos de pontos interiores (veja (Nesterov e Nemirovskii, 1994) para mais detalhes). O próximo problema, que é inteiramente convexo, pode ser visto como uma alternativa mais simples ao problema (24)

$$
\min \operatorname{trace}(V), \quad \text { t.q. } \quad(24 \mathrm{~b}-24 \mathrm{~d})
$$




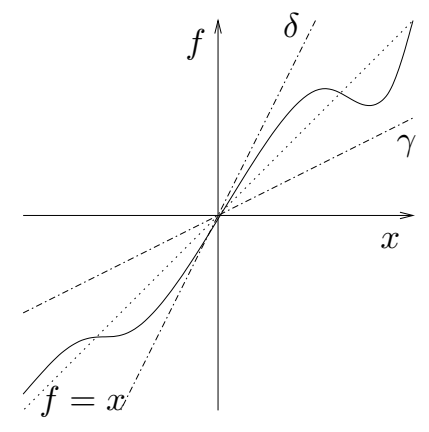

Figura 2: Não linearidade simétrica

A função objetivo reflete a nossa intenção de minimizar a fração $\Gamma=V Q^{-1}$ através da minimização apenas do numerador $V$.

A segunda configuração a ser considerada está ilustrada na Figura 2. Os ângulos entre a reta $f=x$ e as retas $f=\delta x$ e $f=\gamma x$ são idênticos. Com respeito à parametrização do setor $\mathcal{F}(\gamma, \delta)$, isto implica que a relação entre $\gamma$ e $\delta$ deve ser $\gamma=\delta^{-1}$. Portanto, para que possamos aumentar o ângulo de abertura do setor, devemos definir como objetivo a minimização de $\gamma$. Se considerarmos, mais uma vez, que as não linearidades são todas iguais, podemos impor $\Gamma \Delta=\mathbf{I}$ e introduzir a mudança de variáveis

$$
V:=\frac{1}{2}\left(\Gamma+\Gamma^{-1}\right) Q
$$

Como o nosso objetivo é reduzir o valor de $\Gamma$ ou, equivalentemente, aumentar a razão $V Q^{-1}$, podemos lançar mão do problema de otimização

$$
\begin{array}{ll}
\min & \operatorname{trace}(Q-V) \\
& {\left[\begin{array}{cc}
A^{T} P+P A-Q & A^{T} W+V \\
W A+V & -Q
\end{array}\right]<0} \\
& W>0 \\
& V>Q>0, \quad P>0 \quad \text { diagonais. }
\end{array}
$$

Notemos que a solução deste problema é tal que $V Q^{-1}>$ I, o que torna sempre possível recuperar $\Gamma$ a partir de (26). Além disto, $Q-V<0$, o que faz com que a função objetivo tente aumentar $V$ e diminuir $Q$, o que acaba por aumentar a razão $V Q^{-1}$.

Finalmente, consideramos setores na forma $\mathcal{F}(1-$ $\left.\xi_{j}, 1+\xi_{j}\right)$, que estão associados a modelos do tipo FSN (Thygesen e Skelton, 1995). Neste caso, devemos considerar $\Gamma=\mathbf{I}-\Xi, \Delta=\mathbf{I}+\Xi$, em que $\Xi=\left(\xi_{j j}\right)$ é uma matriz diagonal e definida positiva. Assim, temos que $\Gamma+\Delta=2 \mathbf{I}, \Gamma \Delta=\mathbf{I}-\Xi^{2}$, e um possível objetivo pode ser representado como maximizar os limites em $\Xi$. Para este fim, introduzimos a mudança de variáveis

$$
V:=\Xi Q
$$

e o problema de otimização convexo

$$
\begin{array}{ll}
\min & \operatorname{trace}(Q-V) \\
& {\left[\begin{array}{ccc}
A^{T} P+P A-Q & A^{T} W+Q & \mathbf{0} \\
W A+Q & -Q & V \\
\mathbf{0} & V & -Q
\end{array}\right]<0} \\
& \\
& \\
&
\end{array}
$$

Este problema é obtido a partir de manipulações efetuadas em (15) com o auxílio do complemento de Schur. A motivação para a definição da função objetivo é o nosso interesse em maximizar a razão $V Q^{-1}$. Notemos, entretanto, que neste caso $Q-V>0$ (pois $\Xi<\mathbf{I}$ ) e o mínimo valor possível para a função objetivo é zero, caso em que $Q=V$, o que implica $\Xi=\mathbf{I}$.

Como um último comentário: devido à natureza diagonal das matrizes $\Gamma, \Delta$ e $Q$, as formulações definidas acima podem ser facilmente agregadas de modo a refletir diferentes configurações dos setores para um mesmo sistema na forma (1).

\section{EXEMPLO}

Considere o seguinte sistema não linear

$$
\begin{aligned}
& \dot{x}_{1}=x_{1}-x_{3}-h\left(x_{3}\right) \\
& \dot{x}_{2}=-x_{2}-g\left(x_{2}\right)+2 x_{3}+2 h\left(x_{3}\right) \\
& \dot{x}_{2}=5 x_{1}-x_{2}-g\left(x_{2}\right)-2 x_{3}-2 h\left(x_{3}\right)
\end{aligned}
$$

em que as funções $g(\cdot)$ e $h(\cdot)$ estão definidas no conjunto $\mathcal{F}$. Este sistema pode ser reescrito na forma (2) por meio das equações

$$
\left(\begin{array}{l}
\dot{x}_{1} \\
\dot{x}_{2} \\
\dot{x}_{3}
\end{array}\right)=\left[\begin{array}{ccc}
1 & 0 & -1 \\
0 & -1 & 2 \\
5 & -1 & -2
\end{array}\right]\left(\begin{array}{l}
f_{1}\left(x_{1}\right) \\
f_{2}\left(x_{2}\right) \\
f_{3}\left(x_{3}\right)
\end{array}\right),
$$

em que

$$
f(x)=\left(\begin{array}{l}
f_{1}\left(x_{1}\right) \\
f_{2}\left(x_{2}\right) \\
f_{3}\left(x_{3}\right)
\end{array}\right):=\left(\begin{array}{c}
x_{1} \\
x_{2}+g\left(x_{2}\right) \\
x_{3}+h\left(x_{3}\right)
\end{array}\right) .
$$

Se as funções $f_{2}(\cdot)$ e $f_{3}(\cdot)$ pertencem ao conjunto $\mathcal{F}$ então $g(\cdot)$ e $h(\cdot)$ também pertencem a $\mathcal{F}$. Portanto, se a matriz $A$ do sistema (33) for diagonalmente estável, a estabilidade do sistema (30-32) pode ser estabelecida por meio de uma função de Lyapunov do tipo Persidskii. Infelizmente, esta matriz não é diagonalmente estável.

Para que possamos comparar os resultados deste artigo com os métodos baseados no conceito de passividade 
(critérios do círculo e de Popov) disponíveis na literatura reescrevemos o sistema (30-32) na forma de Lur'e

$$
\begin{aligned}
\left(\begin{array}{l}
\dot{x}_{1} \\
\dot{x}_{2} \\
\dot{x}_{3}
\end{array}\right) & =\left[\begin{array}{ccc}
1 & 0 & -1 \\
0 & -1 & 2 \\
5 & -1 & -2
\end{array}\right]\left(\begin{array}{l}
x_{1} \\
x_{2} \\
x_{3}
\end{array}\right)+\left[\begin{array}{cc}
0 & 0 \\
-1 & 2 \\
-1 & -2
\end{array}\right] w \\
y & =-\left[\begin{array}{lll}
0 & 1 & 0 \\
0 & 0 & 1
\end{array}\right]\left(\begin{array}{l}
x_{1} \\
x_{2} \\
x_{3}
\end{array}\right) \\
w & =-\phi(y):=-\left(\begin{array}{l}
g\left(x_{2}\right) \\
h\left(x_{3}\right)
\end{array}\right) .
\end{aligned}
$$

Nesta forma, e levando-se em conta as não linearida$\operatorname{des} g(\cdot)$ e $h(\cdot)$ em $\mathcal{F}$, a estabilidade do sistema (35-37) pode ser avaliada por meio da condição de positividade real. Como a função de transferência entre a entrada $w$ e a saída $y$ do sistema (35-37) não é positiva real estrita (SPR), novamente nenhuma conclusão sobre a estabilidade pode ser obtida. Utilizando versões multivariáveis dos critérios do círculo e de Popov (veja (Khalil, 1996; Haddad e Bernstein, 1993) para mais detalhes) podemos determinar setores

$$
\phi(y)^{T}[\phi(y)-K y] \leq 0
$$

para os quais é possível verificar a estabilidade absoluta. $\mathrm{Na}$ forma mais geral destes resultados o parâmetro do setor $K$ deve ser uma matriz simétrica e definida positiva. Para incertezas escalares, como as tratadas neste artigo, a matriz $K$ deve ser diagonal. Setores na forma (38) podem ser comparados aos setores (8) por meio do escalar

$$
\gamma=\lambda_{\min }(K)
$$

em que $\lambda_{\min }(\cdot)$ denota o autovalor mínimo da matriz $(\cdot)$. Para toda matriz diagonal e definida positiva $K$, verificamos que $\gamma \phi(y)^{T} y \leq \phi(y)^{T} K y$ para todos os vetores $y$. Portanto, voltando a tratar do exemplo, se a origem do sistema (35-37) é um ponto de equilíbrio estável para todas as não linearidades $\phi(\cdot)$ definidas no setor (38), então a origem do sistema (30-32) é também um ponto de equilíbrio estável com relação às não linearidades $g(\cdot)$ e $h(\cdot)$ definidas no setor $\mathcal{F}(0, \gamma)$. Utilizando os resultados do artigo (de Oliveira et al., 2002) podemos calcular o máximo valor do escalar $\gamma$ que pode ser obtido com o critério do círculo $\gamma_{c}=0.14$. Para este exemplo, o máximo valor de $\gamma$ que pode ser obtido com o critério de Popov coincide com o máximo obtido pelo critério do círculo, isto é, $\gamma_{p}=\gamma_{c}=0.14$.

Considerando-se novamente o sistema (33), a especificação original de que os setores associados às não linearidades $g(\cdot)$ e $h(\cdot)$ definidas em (30-32) deveriam estar contidos em $\mathcal{F}$ pode ser exatamente expressa na forma

$$
f_{1}(\cdot) \in \mathcal{F}(1,1), \quad f_{2}(\cdot), f_{3}(\cdot) \in \mathcal{F}(1, \infty) .
$$

Sendo assim, as condições obtidas no presente artigo podem ser utilizadas para tratar diretamente este tipo de setor se definirmos

$$
\Gamma:=\operatorname{diag}(1,1,1), \quad \Delta:=\operatorname{diag}(1,1+\gamma, 1+\gamma) .
$$

Para estas especificações, o Teorema 1 parece indicar que o sistema (30-32) é estável para todo valor positivo de $\gamma$. Soluções factíveis para a LMI (15) podem ser obtidas para valores de $\gamma$ da ordem de $10^{300}$, o que, para todos os efeitos práticos, garante a estabilidade do sistema face às não linearidades $g(\cdot)$ e $h(\cdot)$ em praticamente todo o setor infinito. É difícil verificar numericamente a factibilidade da LMI (15) para valores maiores do que este devido a problemas de precisão numérica. Para nossa surpresa, mesmo o critério puramente quadrático definido no Corolário 6 é capaz de garantir a estabilidade para todo valor de $\gamma$ positivo menor do que $\gamma_{q}=3.01$. Notemos que este valor é aproximadamente vinte e uma vezes maior do que o valor obtido pelos critérios do círculo e de Popov. Portanto, este exemplo ilustra uma situação em que os critérios desenvolvidos neste artigo podem ser bem menos conservadores do que os critérios baseados no conceito de passividade.

Finalmente, a fim de ilustrarmos o uso dos procedimentos de otimização dos setores desenvolvidos na Seção 3, a robustez do sistema (33) será avaliada em relação a perturbações multiplicativas no componente do estado $x_{1}$. O objetivo é determinar o máximo valor de $\xi$ de tal forma que a origem seja um ponto de equilíbrio estável para o sistema (33) cujos setores são definidos por

$$
f_{1}(\cdot) \in \mathcal{F}(1-\xi, 1+\xi), \quad f_{2}(\cdot), f_{3}(\cdot) \in \mathcal{F}(1, \infty) .
$$

Este problema pode ser tratado se introduzirmos uma pequena modificação para que o problema (29) considere apenas a minimização do componente $f_{1}(\cdot)$. O resultado desta otimização provê o valor máximo de $\xi^{*}=0.46$. Isto é, a origem é um ponto de equilíbrio estável do sistema (30-32) para todas as não linearidades $g(\cdot)$ e $h(\cdot)$ em $\mathcal{F}$ e também face a perturbações multiplicativas no primeiro componente do vetor de estado $\left(x_{1}\right)$ limitadas por $\xi^{*}=0.46$.

\section{CONCLUSÕES}

Introduzimos neste artigo um novo teste para estabilidade absoluta de sistemas dinâmicos que apresentam não linearidades no vetor de estados que possam ser descritas por setores. Esta nova condição de estabilidade é expressa na forma de desigualdades matriciais lineares (LMI) e tem como base uma função de Lyapunov do tipo Lur'e. Testes de estabilidade com funções de Lyapunov do tipo Persidskii ou do tipo puramente quadrá- 
tica são gerados como casos particulares, zerando-se alguns elementos da LMI original. Um exemplo numérico demonstra que esta nova condição pode prover resultados menos conservadores do que os obtidos por meio de critérios baseados no conceito de passividade (critérios do círculo e de Popov). Uma análise mais detalhada e criteriosa da relação entra as condições propostas e os critérios clássicos pode ser encontrada em (de Oliveira et al., 2002). Os parâmetros dos setores aparecem explicitamente na LMI e podem ser otimizados utilizando-se técnicas de programação convexa.

\section{AGRADECIMENTOS}

Este trabalho contou com o apóio da FAPESP, da FAPERJ e do CNPq.

\section{REFERÊNCIAS}

Albertini, F. e D'Alessandro, D. (1996). Asymptotic stability of continuous-time systems with saturation nonlinearities, Systems $\&$ Control Letters 29(3): 175-180.

Boyd, S. P., El Ghaoui, L., Feron, E. e Balakrishnan, V. (1994). Linear Matrix Inequalities in System and Control Theory, SIAM, Philadelphia, PA.

Chu, Y.-C. e Glover, K. (1999). Bounds of the induced norm and model reduction errors for systems with repeated scalar nonlinearities, IEEE Transactions on Automatic Control 44(3): 471-483.

Colaneri, P., Geromel, J. C. e Locatelli, A. (1997). Control Theory and Design: An $\mathrm{RH}_{2}-\mathrm{RH}_{\infty}$ viewpoint, Academic Press, San Diego, CA.

de Oliveira, M. C., Geromel, J. C. e Hsu, L. (2002). A new absolute stability test for systems with statedependent perturbations. A ser publicado no International Journal of Robust and Nonlinear Control.

Geromel, J. C., de Oliveira, M. C. e Hsu, L. (1998). LMI characterization of structural and robust stability, Linear Algebra and Its Applications 285(1-3): 6980 .

Haddad, W. M. e Bernstein, D. S. (1993). Explicit construction of quadratic Lyapunov functions for the small gain, positivity, circle, and Popov theorems and their application to robust stability. part I: continuous-time theory, International Journal of Robust and Nonlinear Control 3(4): 313-339.

Hsu, L. e Salgado, L. A. (1980). Structural properties in the stability problem of interconnected systems,
Proceedings of the 2nd IFAC Symposium on Large Scale Systems, pp. 67-77.

Kaszkurewicz, E. e Bhaya, A. (1993). Robust stability and diagonal Lyapunov functions, SIAM Journal on Matrix Analysis and Applications 14: 508-520.

Kaszkurewicz, E. e Bhaya, A. (1994). On a class of globally stable neural circuits, IEEE Transactions on Circuits and Systems - I: fundamental theory and applications 41(2): 171-174.

Kaszkurewicz, E. e Hsu, L. (1979). Stability of nonlinear systems: a structural approach, Automatica 15: 609-614.

Khalil, H. K. (1996). Nonlinear Systems, second edn, Prentice Hall, Inc, Englewood Cliffs, NJ.

Nesterov, Y. E. e Nemirovskii, A. (1994). Interior Point Polynomial Methods in Convex Programming, SIAM, Philadelphia, PA.

Pai, M. A. (1981). Power systems stability, Elsevier, Amsterdan, The Netherlands.

Persidskii, S. K. (1969). Problem on absolute stability, Automation and Remote Control 12: 1889-1895.

Thygesen, U. H. e Skelton, R. E. (1995). Linear systems with finite signal-to-noise ratio: a robustness approach, Proceedings of the 34th IEEE Conference on Decision and Control, pp. 4157-4162. 